\title{
Structured psychiatric assessment schedules - treating the case notes and the patient
}

\author{
AIMS AND METHOD \\ We surveyed the usefulness of a \\ structured method of recording \\ history and mental state examina- \\ tions with a treatment plan in terms \\ of conveying information about \\ diagnosis and management, com- \\ pared with informal methods of \\ recording data. A survey of admission \\ records by nursing and medical raters \\ was followed by introduction of a
}

\author{
standardised assessment format for \\ use by trainees and a re-audit. Initial \\ psychotropic medication was also \\ scrutinised.

RESULTS
The assessment schedule improved
clarity of diagnosis for the medical
and nursing raters alike, but
improvement in management plan
quality was mainly apparent for the

medical rater. The audit also exposed widespread use of hypnotic agents.

\begin{abstract}
CLINICAL IMPLICATIONS
It is suggested that structured assessment schedules facilitate adherence to good standards of clinical practice and may benefit trainees undertaking professional exams as well as having a multi-disciplinary and medico-legal relevance.
\end{abstract}

A high standard of clinical record keeping is a hallmark of good psychiatric practice. Case notes are not just aides-mémoires for doctors but are complex documents that can be used for teaching, research and clinical audit, as well as evidence in the event of litigation. Information obtained when a patient is admitted informs the whole diagnostic and care planning process, including risk management strategies. Psychiatric trainees at our trust presently employ informal methods of clerking patients at admission, using hospital continuation sheets, without adhering to a uniform style. The degree of comprehensiveness of a recorded case history may depend on a variety of factors, not least trainee experience, enthusiasm and the extent to which future involvement with the patient is anticipated. It also follows that admission notes will point towards a diagnosis and impart a clear treatment plan to a greater or lesser extent. We wished to determine in our survey how clinically relevant a structured assessment schedule would be as opposed to present unstructured methods of clerking patients? Did this promote a clearer understanding of diagnosis and management issues as rated by a psychiatrist and a mental health nurse?

\section{The standard}

Incentives for trainees to attain a high standard of documentation of the history and mental state may be provided by the standard required for Royal College of Psychiatrists' (Instructions to candidates available from
The Royal College of Psychiatrists' Examinations Department, 17 Belgrave Sq, London SW1X 8PG) examinations because candidates have been noted to do poorly in clinical examinations when, for example, they stray outside standard classification systems or employ nontechnical terms to describe the diagnosis (Donnelly, 1995). In the day-to-day context, however, absence of key items of information can imply that important aspects of patient management have not been considered and this may have obvious patient care and medicolegal implications (Audit Commission, 1995). Previous local standards of structured documentation within the trust were inspired by the Care Programme Approach. The resultant 16-page assessment form proved unpopular because of its size and had been abandoned for over a year prior to our survey.

\section{The study}

The survey was undertaken within the adult mental health services of Thames Gateway NHS Trust, encompassing the catchment areas of Dartford and Gravesend over a 4-month period and focused on 100 admissions to the acute admissions unit. A structured assessment schedule was designed by the first author (D.L.) in consultation with the psychiatric trainees, a College examiner and the Clinical Audit Committee. It was hypothesised that adherence to the assessment form and the compilation of a management plan would not only document each episode of care but indirectly prove 
beneficial to trainees taking postgraduate examinations, although this was not assessed here.

\section{Method}

Fifty consecutive discharges were subjected to a retrospective case note survey and rated independently by a specialist registrar in psychiatry (D.L.) and a registered mental nurse (M.A.). Criteria were defined and a standard agreed for a rating form that reflected key information that should have been discernible from the admission notes. Each set of admission notes was rated on a fivepoint Likert scale according to their compliance with the criteria (namely agreement or otherwise with the statements) below.

(a) From the admitting officer's notes a likely diagnosis is implied (full or strong agreement implying a formal ICD-10 diagnosis).

(b) A diagnosis is implied 1 week later (full agreement as for first criterion but completion of schedule deferred for up to 1 week as admission data may be unobtainable on first assessment).

(c) From the admitting officer's notes a clear treatment plan is evident (full agreement implying detailed investigations and treatments touching on biological, psychological and social aspects).

(d) From the admitting officer's notes admission to hospital was the most appropriate form of action (full agreement implying significant risk and/or an emergency presentation).

(e) Risk was evaluated and documented in a clear fashion (full agreement implying clear description of risk past and present and the obtaining of collateral information).

(f) Management plan contains clear instructions to militate against risk (full agreement implying clear stating and justification of precautions such as observation level).

Initial prescribed psychotropic medication was also recorded.

A six-page structured assessment form was then introduced, with feedback from trainees - invited to decide which categories of information should be included in a document that was to have utility for emergency and elective admissions alike. The last two pages were the main focus of the audit, where trainees had to fill out the categories of differential diagnosis, investigations and a management section with subcategories embracing physical, biological and social aspects. Trainees were asked to document all initial medication prescribed and to highlight the level of risk (estimated likelihood of self-harm and or violence to others) and measures to address this, such as nursing observations. A further 50 admissions were prospectively rated (also according to the above criteria) in a second audit that was started immediately after introduction of the assessment schedule. Physical examination was not looked at in our survey as it was the subject of a separate trust-wide audit.

\section{Results}

The introduction of the structured assessment schedule improved the imparting of information concerning diagnosis (giving a clear ICD-10 category (World Health Organization, 1992) or indicating a looser description) from $52 \%$ for the medical and nursing raters in audit one to $98 \%$ by the re-audit (see Tables 1 and 2). The clarity of the treatment plan was, for the medical rater (Table 1), facilitated by the use of the form with satisfactory or agree and superlative or strongly agree ratings rising from $34 \%$ to $56 \%$. Risk documentation and amelioration for the medical rater improved from $36 \%$ and $78 \%$, respectively, (also taking agree and strongly agree ratings together) to $52 \%$ and $90 \%$, respectively, with use of the schedule. The nursing rater's estimation of the clarity of the treatment plans was not enhanced by the use of the schedule, nor was the documenting of risk (Table 2). The overall appreciation of measures to counteract risk rose by only $2 \%$, although superlative scores from the nurse rater climbed by $12 \%$ when the schedule was employed.

The prescribing of 'as required' zopiclone was found to be widespread, with $57 \%$ of all acute admissions being charted for this agent. Lorazepam on an 'as required basis' was charted for $28 \%$ of all admissions and like the hypnotics no attempt was made by any trainee to time limit this prescription, although individual doses had been restricted to a maximum amount in a given 24 -hour period.

\section{Comment and clinical implications}

We believe that use of a structured assessment schedule has encouraged trainees to commit themselves further in stating a likely diagnosis for a given admission as well as outlining a more detailed management plan that was described by informal methods of clerking admissions. Consequently those reviewing case notes are likely (almost twice as likely for diagnosis in our survey) to have a clearer understanding of these issues. Rates of completion of other data categories in the schedule were not studied here and it was left to consultants within individual teams to ensure accuracy was maintained in documenting, for example, mental state examination findings. Such consultant-led supervision of records has been previously shown to be useful not only for verification of clinical findings but also in improving standards of data recording (Kareem \& Ashby, 2000). The schedule may help to bridge the gap between professional exams and day-to-day clinical practice as the categories we audited are relevant for any clinical postgraduate examination and also reflect a high standard of patient care.

The schedule allowed for greater comprehensibility by the medical, as opposed to the nursing, rater of the treatment plans outlined, but this does not necessarily undermine its relevance in a multi-disciplinary context. Nursing staff may look for different aspects of treatment plans in judging the comprehensiveness of admissions records. Possible methodological limitations include the absence of rater blindness to the purpose of the original papers 
Table 1. Doctors' comparison - before and after introduction of structured assessment form

original

papers

\begin{tabular}{|c|c|c|c|c|c|c|}
\hline \multirow[b]{2}{*}{ Doctors' ratings } & \multicolumn{2}{|c|}{ Disagree (\%) } & \multicolumn{2}{|c|}{$\begin{array}{l}\text { No view/ } \\
\text { ambiguous (\%) }\end{array}$} & \multicolumn{2}{|c|}{ Agree (\%) } \\
\hline & Before & After & Before & After & Before & After \\
\hline From admitting officers' notes - a likely diagnosis is implied & 14 & 2 & 34 & 0 & 52 & 98 \\
\hline A diagnosis is implied 1 week later & 4 & 0 & 26 & 2 & 70 & 98 \\
\hline Risk was evaluated and documented in a clear fashion & 16 & 12 & 48 & 36 & 36 & 52 \\
\hline Management plan contains clear instructions to militate against risk & 12 & 6 & 10 & 4 & 78 & 90 \\
\hline
\end{tabular}

Table 2. Nurses' comparison - before and after introduction of structured assessment form

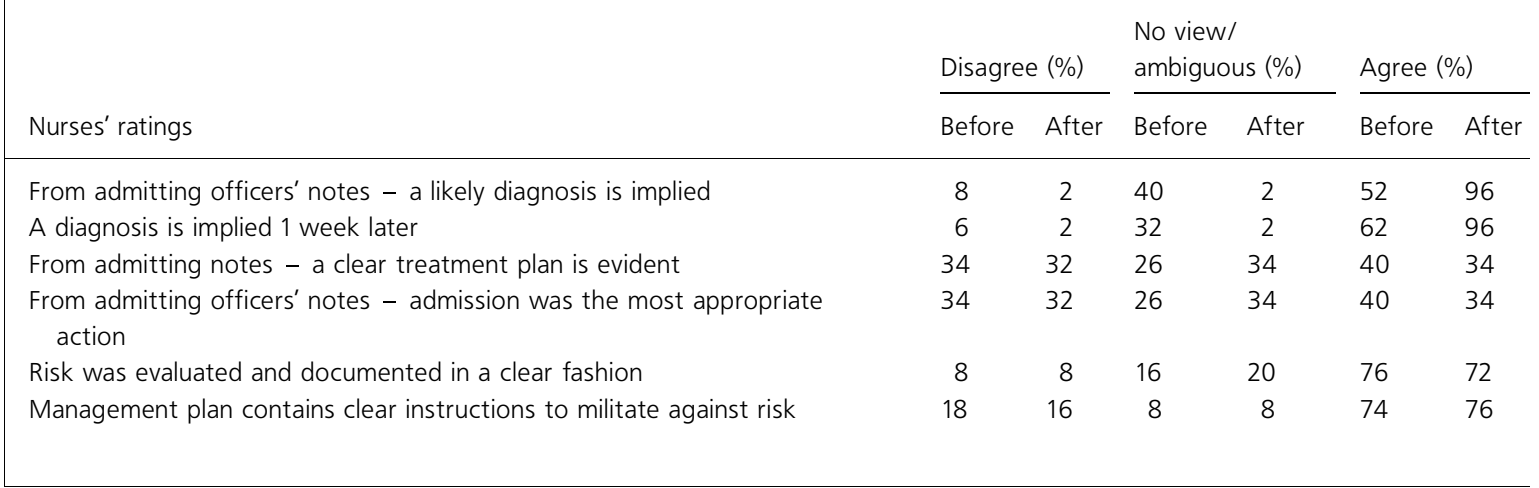

study, which could partially explain the disparity between the two raters. The overall changes highlighted after introduction of the schedule could also be attributed to the audit process. The process of clinical audit can by itself lead to improvements in standards of documentation (O'Hare 1995) but in the face of regular staff turnover may not harness durable quality assurance. The trainees were not informed of the second audit to minimise the effect of the audit process and it is also significant that a changeover of junior doctors occurred before the second audit began.

Omissions have been shown to be less frequent in consultation notes delineating data categories (Small \& Fawzy, 1988), however, completion rates of the treatment plan section of our form need to be improved. Almost half of the treatment plans were still not being documented in sufficient detail. Further audit will be required to establish if the improvements we have highlighted can be built upon as trainees become accustomed to using the schedule. The widespread use of hypnotics has caused concern and led to an immediate separate audit but the primary finding of our study is the preliminary evidence of the usefulness of structured assessment schedules, the discrepancy between nursing and medical staff views of the completeness of treatment plans being a useful avenue for further scrutiny.

\section{Acknowledgements}

We wish to thank John Wilkins and Sue Whitehead of the Clinical Audit Department of Thames Gateway NHS Trust for their advice and assistance.

Copies of the assessment schedule employed in the survey are available on request from D. Lyons, Brixton Community Mental Health Team, 308-312 Brixton Road, London SW9 6AA.

\section{References}

AUDIT COMMISSION (1995) Setting the Record Straight, A Study of Hospital Medical Records. The Audit Commission: London.

DONNELLY, P. (1995) Why trainees fail mock MRCPsych clinical examinations. Psychiatric Bulletin, 19, 302-304

KAREEM, O. S. \& ASHBY, C. A. (2000) Mental state examinations by psychiatric trainees in a community NHS trust. The importance of a standardised format. Psychiatric Bulletin, 24, 109-110.

O'HARE,T. (1995) Improvements in practice from assessing standards of recording psychiatric case-notes. Psychiatric Bulletin, 19, 352-354.

SMALL, G. \& FAWZY, F. (1988) Data omitted from psychiatric consultation notes. Journal of Clinical Psychiatry, 49, 307-309.

WORLD HEALTH ORGANIZATION (1992) The ICD-10 Classification of Mental and Behavioural Disorders. Geneva:WHO.

${ }^{*}$ Declan Lyons Specialist Registrar, Brixton Community Mental HealthTeam, 308-312 Brixton Road, London SW9 6AA, Mark Amos Mental Health Nurse, V. M. Mathew Professor and Consultant Psychiatrist, Thames Gateway NHS Trust, The Little Brook Hospital, Archery Lane, Stone, Dartford, Kent DA2 6AU 